Prons

trobertivier

Journal of Nonlinear Mathematical Physics

ISSN (Online): 1776-0852 ISSN (Print): 1402-9251

$P^{\text {rrumisis }}$ Journal Home Page: https://www.atlantis-press.com/journals/jnmp

\title{
Geometry of rank 2 distributions with nonzero Wilczynski invariants*
}

Boris Doubrov, Igor Zelenko

To cite this article: Boris Doubrov, Igor Zelenko (2014) Geometry of rank 2 distributions with nonzero Wilczynski invariants*, Journal of Nonlinear Mathematical Physics 21:2, 166-187, DOI: https://doi.org/10.1080/14029251.2014.900985

To link to this article: https://doi.org/10.1080/14029251.2014.900985

Published online: 04 January 2021 


\title{
Geometry of rank 2 distributions with nonzero Wilczynski invariants*
}

\author{
Boris Doubrov \\ Department of Mathematical Physics, Belarussian State University \\ Nezavisimosti Ave. 4, Minsk 220050, Belarus \\ doubrov@islc.org \\ Igor Zelenko \\ Department of Mathematics, Texas A\&M University \\ College Station, TX 77843-3368, USA \\ zelenko@math.tamu.edu
}

Received 27 August 2013

Accepted 30 December 2013

\begin{abstract}
In the famous 1910 "cinq variables" paper Cartan showed in particular that for maximally nonholonomic rank 2 distributions in $\mathbb{R}^{5}$ with non-zero covariant binary biquadratic form the dimension of the pseudo-group of local symmetries does not exceed 7 and among such distributions he described the one-parametric family of distributions for which this pseudo-group is exactly 7-dimensional. Using the novel interpretation of the Cartan covariant binary biquadratic form via the classical Wilczynski invariant of curves in projective spaces associated with abnormal extremals of the distributions $[4,27,28]$ one can generalize this Cartan result to rank 2 distributions in $\mathbb{R}^{n}$ satisfying certain genericity assumption, called maximality of class, for arbitrary $n \geq 5$.

In the present paper for any rank 2 distribution of maximal class with at least one nonvanishing generalized Wilczynski invariants we construct the canonical frame on a $(2 n-3)$-dimensional bundle and describe explicitly the moduli spaces of the most symmetric models. The relation of our results to the divergence equivalence of Lagrangians of higher order is given as well.
\end{abstract}

Keywords: Abnormal extremals of distributions; self-dual curves in projective space; Wilczynski invariants; canonical frames; $\mathfrak{s l}_{2}$-representations.

2010 Mathematics Subject Classification: 58A30, 53A55, 34C14.

\section{Introduction}

A rank 2 distribution on an $n$-dimensional manifold $M$ or shortly $(2, n)$-distribution is a rank 2 subbundle of the tangent bundle $T M$ or a field of planes on $M$. We are interested in the local equivalence problem for these geometric structures under the natural action of the group of germs of diffeomorphisms of $M$. Starting from the dimension $n=5$ this equivalence problem is non-trivial for generic objects, i.e. generic rank 2 distributions have functional differential invariants.

The case $n=5$ was treated by Èlie Cartan in his famous paper [8]. First, starting from any maximally nonholonomic $(2,5)$-distribution he constructed a canonical coframe on a bundle of dimension 14 over $M$. In particular, this implies that the dimension of the pseudo-group of local symmetries of such distributions does not exceed 14. Second, Cartan constructed the fundamental invariant of the distribution, which is a special homogeneous polynomial of degree 4 on each fiber of the distribution. We refer to it as Cartan tensor. In particular, he has shown that there exists exactly one distribution, up to a local equivalence, such that its Cartan tensor vanishes identically. This distribution is locally equivalent to the distribution, associated with the underdetermined ODE

${ }^{*}$ Dedicated to Andrei Agrachev on the occasion of his 60 th birthday 
$z^{\prime}(x)=\left(y^{\prime \prime}(x)\right)^{2}$. Moreover, this distribution is the unique distribution, up to a local equivalence, having 14 dimensional pseudo-group of local symmetries. Besides, from Cartan's analysis it follows that if the Cartan tensor does not vanish then the pseudo-group of local symmetries of a maximally nonholonomic $(2,5)$-distribution does not exceed 7 . The family of all distributions for which this pseudo-group is 7-dimensional can be explicitly described and depends on one constant.

In [28] the second author found an alternative interpretation of the Cartan tensor exploiting the notion of abnormal extremals of distributions (see section 2). Namely, to any abnormal extremal $\gamma$ of a rank 2 distribution in $\mathbb{R}^{n}$ with $n \geq 5$ one can assign a curve of Lagrangian subspaces in a linear symplectic space (of dimension $2(n-3)$ ), i.e. a curve in a Lagrangian Grassmannian. This curve is obtained via the linearization of the flow of abnormal extremals along the extremal $\gamma$ and is called a Jacobi curve by analogy with Jacobi fields in the classical Calculus of Variations. For $n \geq 5$ the differential geometry of curves in Lagrangian Grassmannians (under the natural action of the linear symplectic group) is non-trivial.

The invariants of curves in Lagrangian Grassmannains can be obtained in several ways. One way is to use the notion of the cross-ratio of four points on the curve, generalizing the classical notion of the cross-ration of four points of the projective lines. In [3, 27] the asymptotic of the cross-ratio of four points on the curve in Lagrangian Grassmannian near the diagonal was studied. It turns out that for $n=5$ the first non-trivial differential invariant of Jacobi curves (from the aforementioned asymptotic) coincides, after an appropriate interpretation, with the Cartan tensor ( [28]) and therefore for $n>5$ it gives a natural generalization of this tensor ( [27]).

Moreover, in the case of $(2, n)$ - distributions, the geometry of Jacobi curves can be reduced to the geometry of the so-called self-dual curves in projective spaces (of dimension $2 n-7$ ) by a series of osculations together with the operation of taking skew symmetric complements $[4,27]$. The differential geometry of curves in projective spaces goes back to E.J. Wilczynski, who constructed in 1906 the fundamental set of (relative) invariants [25]: if $k$ is the dimension of the projective space then for a non-degenerate curve in the projective space the set of fundamental invariants consists of $k-1$ relative invariants $\mathscr{W}_{i}$ of degree $i+2, i=1, \ldots, k-1$, called the Wilczynski invariants. He has also shown that the curve is self-dual if and only if all invariants of odd degree vanish. Since $k=2 n-7$ for a $(2, n)$-distribution, we get $(n-4)$ nontrivial invariants of this distribution coming from the Wilczynski invariants of even degree of the corresponding self-dual curves in projective spaces. These invariants of the distribution are called the generalized Wilczynski invariants of rank 2 distributions (see section 5 for details). For $n=5$ the Cartan tensor coincides with the generalized Wilczynski invariant of degree 4 (which is the unique generalized Wilczynski invariant in this case).

Further, this approach was used in $[10,11]$ for constructing the canonical frames for rank 2 distributions on manifolds of dimension $n>5$ under very mild genericity assumptions called maximality of class. This construction needs nothing more than some simple facts from the classical theory of curves in projective spaces such as the existence of the canonical projective structure on such curves, i.e. a special set of parametrizations defined up to a Möbius transformation (see section 5 below). The canonical frame for such distributions is constructed in a unified way on a bundle of total dimension $2 n-1$. Remarkably, this construction is independent of the nilpotent approximation (the Tanaka symbol $[24,30]$ ) of a distribution at a point and even independent of its small growth vector.

The dimension of the bundle with the canonical frame cannot be reduced in general, because there exists a unique, up to a local equivalence, rank 2 distribution of maximal class in $\mathbb{R}^{n}, n>5$ 
with the pseudo-group of local symmetries of dimension equal to $2 n-1$ ( $[10,11])$. This distribution is locally equivalent to the distribution, associated with the underdetermined (Monge) ODE $z^{\prime}(x)=$ $\left(y^{(n-3)}(x)\right)^{2}$ (see also [7], where the same is proved for more restrictive class of rank 2 distributions associated with Monge equations). Besides, for this most symmetric rank 2 distribution of maximal class all generalized Wilczynski invariants vanish identically.

However, if we assume that at least one generalized Wilczynski invariant does not vanish, then one would expect that the canonical frame can be constructed on a bundle of smaller dimension. In the present paper we show that in this situation the canonical frame can be constructed in a unified way on a bundle of dimension $2 n-3$ for all $n \geq 5$ (Theorem 7.1, section 7), i.e. a gap phenomenon occurs (namely, the dimension of the pseudo-group of symmetries drops more than by 1 compared to the most symmetric model without the restriction on the generalized Wilczynski invariants) similar to one observed in $[18,19]$. We also describe all models with the pseudo-group of local symmetries of dimension $2 n-3$, i.e. the most symmetric ones, among the considered class of objects (Theorem 1.1 below and its reformulation in Theorem 9.1, section 9).

The key point is that in the considered case the canonical parametrization, up to a shift, can be distinguished on abnormal extremals instead of the canonical projective structure. Note that the same technique both for the construction of canonical frames and for the analysis of the most symmetric models was used in our recent paper [16] for rank 2 distributions of maximal class without restrictions on the generalized Wilczynski invariants but with distinguished affine subdistributions or more general control system with one input on them. In this case the additional structures on the distribution impose the distinguished parametrization, up to a shift, on abnormal extremals as well. However, the class of equivalence problems considered in the present paper is more complicated than in [16]. For example, the description of the moduli spaces of the most symmetric models is more involved and based on some new features of the geometry of linear differential equations.

Let us describe the most symmetric rank 2 distributions with at least one non-vanishing Wilczynski invariant in more detail. Given a tuple of $n-3$ real constants $\left(r_{1}, \ldots, r_{n-3}\right)$, let $D_{\left(r_{1}, \ldots, r_{n-3}\right)}$ be the distribution associated with the following underdetermined ordinary differential equation (Monge equation):

$$
z^{\prime}(x)=\left(y^{(n-3)}(x)\right)^{2}+r_{1}\left(y^{(n-4)}(x)\right)^{2}+\ldots r_{n-3} y^{2}(x) .
$$

More explicitly, it can be defined as the distribution in $R^{n}$, with coordinates $\left(x, y_{0}, \ldots, y_{n-3}, z\right)$, spanned by the following vector fields:

$$
\begin{aligned}
X_{1}= & \frac{\partial}{\partial x}+y_{1} \frac{\partial}{\partial y_{0}}+\cdots+y_{n-3} \frac{\partial}{\partial y_{n-4}}+ \\
& \left(y_{n-3}^{2}+r_{1} y_{n-4}^{2}+r_{2} y_{n-5}^{2}+\ldots r_{n-3} y_{0}^{2}\right) \frac{\partial}{\partial z}, \\
X_{2} & =\frac{\partial}{\partial y_{n-3}} .
\end{aligned}
$$

Let $\mathscr{D}_{n}=\left\{D_{r_{1}, \ldots, r_{n-3}}:\left(r_{1}, \ldots, r_{n-3}\right) \in \mathbb{R}^{n-3}\right\}$ be the family of all such distributions. There is a special 1 -foliation $\mathscr{F}$ (i.e. a foliation by curves) in $\mathscr{D}_{n}$ with a singularity at the origin (under the identification of $\mathscr{D}_{n}$ with $\mathbb{R}^{n-3}$ ) such that the rank 2 distributions from the same leaf of $\mathscr{F}$ are locally equivalent and the rank 2 distributions from different leaves of $\mathscr{F}$ are not equivalent. Among all leaves of $\mathscr{F}$ there is an exceptional leaf $\mathscr{F}(0)$ passing through the origin. The rank 2 distributions from this leaf are locally equivalent to the most symmetric rank 2 distribution in $\mathbb{R}^{n}$ of maximal 
class. It turns out that the space of the most symmetric rank 2 distributions of maximal class in $\mathbb{R}^{n}$ with nonzero Wilczynski invariants can be identified with the quotient space of $\mathscr{D}_{n} \backslash \mathscr{F}(0)$ by the foliation $\mathscr{F}$.

In more details, first, as shown in $[10,11]$, any most symmetric rank 2 distribution in $\mathbb{R}^{n}$ of maximal class with $n \geq 5$ is locally equivalent to $D_{(0, \ldots, 0)}$. Next, among all distributions $D_{\left(r_{1}, \ldots, r_{n-3}\right)}$ there is a one-parametric family of distributions locally equivalent to $D_{(0, \ldots, 0)}$. To describe this family we need the following definition

Definition 1.1. The tuple of $m$ numbers $\left(r_{1}, \ldots, r_{m}\right)$ is called exceptional if the roots of the polynomial

$$
\lambda^{2 m}+\sum_{i=1}^{m}(-1)^{i} r_{i} \lambda^{2(m-i)}
$$

constitute an arithmetic progression (with the zero sum in this case). Equivalently, $\left(r_{1}, \ldots, r_{m}\right)$ is exceptional if $r_{i}=\alpha_{m, i}\left(\frac{r_{1}}{\alpha_{m, 1}}\right)^{i}, 1 \leq i \leq m$, where the constants $\alpha_{m, i}, 1 \leq i \leq m$, satisfy the following identity

$$
x^{2 m}+\sum_{i=1}^{m}(-1)^{i} \alpha_{m, i} x^{2(m-i)}=\prod_{i=1}^{m}\left(x^{2}-(2 i-1)^{2}\right) .
$$

We prove (Corollary 8.1, section 8 ) that the distribution $D_{\left(r_{1}, \ldots, r_{n-3}\right)}$ is locally equivalent to the distribution $D_{(0, \ldots, 0)}$ (or, equivalently, has the algebra of infinitesimal symmetries of the maximal possible dimension among all rank 2 distributions of maximal class in $\mathbb{R}^{n}$ ) if and only if the tuple $\left(r_{1}, \ldots, r_{n-3}\right)$ is exceptional in the sense of Definition 1.1. As far as we know, this simple but nice observation was not mentioned in the existing literature. This observation is based on the following simple fact from the representation theory of the Lie algebra $\mathfrak{s l}_{2}:$ the spectrum of any element in the image of an irreducible representation of $\mathfrak{s l}_{2}$ forms an arithmetic progression (see Proposition 8.1, section 8). We have the following

Theorem 1.1. If a rank 2 distribution in $\mathbb{R}^{n}$ of maximal class, $n \geq 5$, with at least one non-vanishing generalized Wilczynski invariant has a group of local symmetries of dimension $2 n-3$, then it is locally equivalent to the distribution $D_{\left(r_{1}, \ldots, r_{n-3}\right)}$, where the tuple $\left(r_{1}, \ldots, r_{n-3}\right)$ is not exceptional. Two distributions $D_{\left(r_{1}, \ldots, r_{n-3}\right)}$ and $D_{\left(\tilde{r}_{1}, \ldots, \tilde{r}_{n-3}\right)}$ are locally equivalent if and only if

$$
\text { there exists } c \neq 0 \text { such that } \tilde{r}_{i}=c^{2 i} r_{i}, \quad 1 \leq i \leq n-3 \text {. }
$$

The aforementioned foliation $\mathscr{F}$ on the space $\mathscr{D}_{n}$ can be described as follows: the exceptional leaf $\mathscr{F}(0)$ consists of the exceptional tuples in the sense of Definition 1.1; other leaves are exactly the equivalence classes on $\mathscr{D}_{n} \backslash \mathscr{F}(0)$ with respect to the equivalence relation given by (1.6). Note that the exceptional leaf $\mathscr{F}(0)$ is also the union of three equivalence classes with respect to the same equivalence relation, one of which is the origin.

The family of distributions $\mathscr{D}_{n}$ is also related with the Lagrangians

$$
\int\left(\left(y^{(n-3)}(x)\right)^{2}+r_{1}\left(y^{(n-4)}(x)\right)^{2}+\ldots r_{n-3} y^{2}(x)\right) d x,
$$

which are quadratic with respect to the derivatives and have constant coefficients. It is well known (see [23], discussion in the beginning of p. 242 there) that these Lagrangians are the most symmetric 
ones among all Lagrangians

$$
\int F\left(x, y(x), \ldots, y^{(n-3)}(x)\right) d x
$$

with $F_{y^{(n-3)} y^{(n-3)}} \neq 0$, up to a contact transformations and modulo divergence. Our results here together with the relation of this equivalence problem and its modification to the equivalence of very special rank 2 distributions of maximal class studied in [12] give an alternative proof of this fact. Note that for these most symmetric Lagrangians the Euler-Lagrange equation is a linear equation with constant coefficients (such that its characteristic polynomial coincides with the polynomial in (1.4) where $m=n-3$ ).

Note that for rank 2 distributions in $\mathbb{R}^{5}$ the notion of maximality of class coincides with the condition that the small growth vector is equal to $(2,3,5)$. As was shown by Èlie Cartan in his famous paper [8] the most symmetric distribution among all distributions with small growth vector $(2,3,5)$ has 14 dimensional algebra of infinitesimal symmetries and this distribution is the unique distribution with identically zero Cartan invariant (which coincides with the (unique in this case) generalized Wilczynski invariant). Therefore in this case our approach gives the unified construction of the canonical frame on a 7-dimensional bundle for all distributions with the small growth vector $(2,3,5)$ except the most symmetric one, which provides also an alternative way to get the Cartan classification of submaximal symmetric models for these distributions [8, chapter IX]. Note also that in the case $n=5$ the construction of the canonical frame was already done in the $\mathrm{PhD}$ thesis of the second author [31, subsection 10.5]. It is worth to mention that for $n=5$ an alternative way to describe these submaximal models is via the family of underdetermined ODEs (Monge equations) $z^{\prime}(x)=\left(y^{\prime \prime}(x)\right)^{\alpha}$ with $\alpha \notin\left\{-1, \frac{1}{3}, \frac{2}{3}, 2\right\}$ and one additional equation $z^{\prime}(x)=\ln y^{\prime \prime}(x)$ (see, for example, [22, Example 6], [18, section 5]).

In the case $n>5$ the question whether the most symmetric distribution of maximal class is the only distribution within this class with all vanishing generalized Wilczynski invariants remains open. It is known however that this is true in the class of distributions associated with Monge equations:

$$
z^{\prime}=F\left(x, y(x), \ldots, y^{(n-3)}(x)\right)
$$

with the right hand side independent of $z(x)$ and non-linear in $y^{(n-3)}([12])$. In other words, it was shown there that the vanishing of all generalized Wilczynski invariants for the distribution associated with the Monge equation (1.8) implies that this distribution is equivalent to the most symmetric one.

The paper is organized as follows. The main results are given in sections 7 and 9 (Theorem 7.1 and Theorem 9.1, which is the reformulation of Theorems 1.1 above). Note that the proofs of Theorem 7.1 and Theorem 9.1 are essentially the repetitions of the proofs of Theorem 3 and Theorem 4 from [16], respectively, modified appropriately to the equivalence problem considered in the present paper. They are given for the sake of completeness and self-containment of the exposition. Sections 2-6 are preparatory for section 7, section 8 is preparatory for section 9. In sections 2-5 we list all necessary facts about abnormal extremals of rank 2 distributions, their Jacobi curves and the invariants of unparametrized curves in projective spaces. The details can be found in $[10,26,27]$. In section 6 we summarize the main results of $[10,11]$ about canonical frames for rank 2 distributions 
of maximal class in order to compare them with the analogous results of sections 7 and 9 . In section 8 we list all necessary facts about the invariants of parametrized self-dual curves in projective spaces.

\section{Abnormal extremals of rank 2 distributions}

Let $D$ be a rank 2 distribution on a manifold $M$. A smooth section of a vector bundle $D$ is called a horizontal vector field of $D$. Taking iterative brackets of horizontal vector fields of $D$, we obtain the natural filtration $\left\{\operatorname{dim} D^{j}(q)\right\}_{j \in \mathbb{N}}$ on each tangent space $T_{q} M$. Here $D^{j}$ is the $j$-th power of the distribution $D$, i.e., $D^{j}=D^{j-1}+\left[D, D^{j-1}\right], D^{1}=D$, or , equivalently, $D^{j}(q)$ is a linear span of all Lie brackets of the length not greater than $j$ of horizontal vector fields of $D$ evaluated at $q$.

Assume that $\operatorname{dim} D^{2}(q)=3$ and $\operatorname{dim} D^{3}(q)>3$ for any $q \in M$. Denote by $\left(D^{j}\right)^{\perp} \subset T^{*} M$ the annihilator of the $j$ th power $D^{j}$, namely

$$
\left(D^{j}\right)^{\perp}=\left\{(p, q) \in T^{*} M: p \cdot v=0 \forall v \in D^{j}(q)\right\} .
$$

Recall that abnormal extremals of $D$ are by definition the Pontryagin extremals with the vanishing Lagrange multiplier near the functional for any extremal problem with constrains, given by the distribution $D$. They depend only on the distribution $D$ and not on a functional.

It is easy to show (see, for example, $[11,26]$ ) that for rank 2 distributions all abnormal extremals lie in $\left(D^{2}\right)^{\perp}$ and that through any point of the codimension 3 submanifold $\left(D^{2}\right)^{\perp} \backslash\left(D^{3}\right)^{\perp}$ of $T^{*} M$ passes exactly one abnormal extremal or, in other words, $\left(D^{2}\right)^{\perp} \backslash\left(D^{3}\right)^{\perp}$ is foliated by the characteristic 1-foliation of abnormal extremals. To describe this foliation let $\pi: T^{*} M \mapsto M$ be the canonical projection. For any $\lambda \in T^{*} M, \lambda=(p, q), q \in M, p \in T_{q}^{*} M$, let $\mathfrak{s}(\lambda)(\cdot)=p\left(\pi_{*} \cdot\right)$ be the canonical Liouville form and $\sigma=d \mathfrak{s}$ be the standard symplectic structure on $T^{*} M$. Since the submanifold $\left(D^{2}\right)^{\perp}$ has odd codimension in $T^{*} M$, the kernels of the restriction $\left.\sigma\right|_{\left(D^{2}\right)^{\perp}}$ of $\sigma$ on $\left(D^{2}\right)^{\perp}$ are not trivial. At the the points of $\left(D^{2}\right)^{\perp} \backslash\left(D^{3}\right)^{\perp}$ these kernels are one-dimensional. They form the characteristic line distribution in $\left(D^{2}\right)^{\perp} \backslash\left(D^{3}\right)^{\perp}$, which will be denoted by $\mathscr{C}$. The line distribution $\mathscr{C}$ defines the desired characteristic 1-foliation on $\left(D^{2}\right)^{\perp} \backslash\left(D^{3}\right)^{\perp}$ and the leaf of this foliation through a point is exactly the abnormal extremal passing through this point. From now on we shall work with abnormal extremals which are integral curves of the characteristic distribution $\mathscr{C}$.

The characteristic line distribution $\mathscr{C}$ can be easily described in terms of a local basis of the distribution $D$, i.e. 2 horizontal vector fields $X_{1}$ and $X_{2}$ such that $D(q)=\operatorname{span}\left\{X_{1}(q), X_{2}(q)\right\}$ for all $q$ from some open set of $M$. Denote by

$$
X_{3}=\left[X_{1}, X_{2}\right], X_{4}=\left[X_{1},\left[X_{1}, X_{2}\right]\right], X_{5}=\left[X_{2},\left[X_{1}, X_{2}\right]\right] .
$$

Let us introduce the "quasi-impulses" $u_{i}: T^{*} M \mapsto \mathbb{R}, 1 \leq i \leq 5$,

$$
u_{i}(\lambda)=p \cdot X_{i}(q), \lambda=(p, q), q \in M, p \in T_{q}^{*} M .
$$

Then by the definition

$$
\left(D^{2}\right)^{\perp}=\left\{\lambda \in T^{*} M: u_{1}(\lambda)=u_{2}(\lambda)=u_{3}(\lambda)=0\right\} .
$$

As usual, for a given function $h: T^{*} M \mapsto \mathbb{R}$ denote by $\vec{h}$ the corresponding Hamiltonian vector field defined by the relation $i_{\vec{h}} \sigma=-d h$. Then by the direct computations (see, for example, [11]) the characteristic line distribution $\mathscr{C}$ satisfies

$$
\mathscr{C}=\operatorname{span}\left\{\mathrm{u}_{4} \vec{u}_{2}-\mathrm{u}_{5} \vec{u}_{1}\right\}
$$




\section{Jacobi curves of abnormal extremals}

Now we are ready to define the Jacobi curve of an abnormal extremal of $D$. For this first lift the distribution $D$ to $\left(D^{2}\right)^{\perp}$, namely considered the distribution $\mathscr{J}$ on $\left(D^{2}\right)^{\perp}$ such that

$$
\mathscr{J}(\lambda)=\left\{v \in T_{\lambda}\left(D^{2}\right)^{\perp}: d \pi(v) \in D(\pi(\lambda))\right\} .
$$

Note that $\operatorname{dim} \mathscr{J}=n-1$ and $\mathscr{C} \subset \mathscr{J}$ by (2.4). The distribution $\mathscr{J}$ is called the lift of the distribution $D$ to $\left(D^{2}\right)^{\perp} \backslash\left(D^{3}\right)^{\perp}$.

Given a segment $\gamma$ of an abnormal extremal (i.e. of a leaf of the 1-characteristic foliation) of $D$, take a sufficiently small neighborhood $O_{\gamma}$ of $\gamma$ in $\left(D^{2}\right)^{\perp}$ such that the quotient $N=$ $O_{\gamma} /$ (the characteristic one-foliation) is a well defined smooth manifold. The quotient manifold $N$ is a symplectic manifold endowed with the symplectic structure $\bar{\sigma}$ induced by $\left.\sigma\right|_{\left(D^{2}\right)^{\perp}}$. Let

$$
\phi: O_{\gamma} \rightarrow N
$$

be the canonical projection on the factor. Define the following curves of subspaces in $T_{\gamma} N$ :

$$
\lambda \mapsto \phi_{*}(\mathscr{J}(\lambda)), \quad \forall \lambda \in \gamma .
$$

Informally speaking, these curves describe the dynamics of the distribution $\mathscr{J}$ w.r.t. the characteristic 1-foliation along the abnormal extremal $\gamma$. Note that if we choose another neighborhood $\widetilde{O}_{\gamma}$ of $\gamma$ with the same properties, then the corresponding quotient map coincides with the map $\phi$ on $O_{\gamma} \cap \widetilde{O}_{\gamma}$. This implies that the curve defined by (3.3) is independent of the choice of the neighborhood $O_{\gamma}$, because the only data about the quotient map $\phi$ that appears there is the differentials of $\phi$ at points of $\gamma$.

Note that there exists a straight line, which is common to all subspaces appearing in (3.3) for any $\lambda \in \gamma$. So, it is more convenient to get rid of it by a factorization. Indeed, let $e$ be the Euler field on $T^{*} M$, i.e., the infinitesimal generator of homotheties on the fibers of $T^{*} M$. Since a transformation of $T^{*} M$, which is a homothety on each fiber with the same homothety coefficient, sends abnormal extremals to abnormal extremals, we see that the vector $\bar{e}=\phi_{*} e(\lambda)$ is the same for any $\lambda \in \gamma$ and lies in any subspace appearing in (3.3). Let

$$
J_{\gamma}(\lambda)=\phi_{*}(\mathscr{J}(\lambda)) /\{\mathbb{R} \bar{e}\}, \quad \forall \lambda \in \gamma
$$

The (unparametrized) curve $\lambda \mapsto J_{\gamma}(\lambda), \lambda \in \gamma$ is called the Jacobi curve of the abnormal extremal $\gamma$. It is clear that all subspaces appearing in (3.4) belong to the space

$$
W_{\gamma}=\left\{v \in T_{\gamma} N: \bar{\sigma}(v, \bar{e})=0\right\} /\{\mathbb{R} \bar{e}\} .
$$

and that

$$
\operatorname{dim} J_{\gamma}(\lambda)=n-3
$$

The space $W_{\gamma}$ is endowed with the natural symplectic structure $\tilde{\sigma}_{\gamma}$ induced by $\bar{\sigma}$. Also $\operatorname{dim} W_{\gamma}=$ $2(n-3)$.

Given a subspace $L$ of $W_{\gamma}$ denote by $L^{<}$the skew-orthogonal complement of $L$ with respect to the symplectic form $\tilde{\sigma}_{\gamma}, L^{L}=\left\{v \in W_{\gamma}, \sigma_{\gamma}(v, \ell)=0 \quad \forall \ell \in L\right\}$. Recall that the subspace $L$ is called isotropic if $L \subseteq L^{<}$, coisotropic if $L^{\angle} \subseteq L$, and Lagrangian, if $L=L^{\angle}$. Directly from the definition, 
the dimension of an isotropic subspace does not exceed $\frac{1}{2} \operatorname{dim} W_{\gamma}$, and a Lagrangian subspace is an isotropic subspace of the maximal possible dimension $\frac{1}{2} \operatorname{dim} W_{\gamma}$. The set of all Lagrangian subspaces of $W_{\gamma}$ is called the Lagrangian Grassmannian of $W_{\gamma}$.

It is easy to see $([11,27])$ that the Jacobi curve of an abnormal extremal consists of Lagrangian subspaces, i.e. it is a curve in the Lagrangian Grassmannian of $W_{\gamma}$. In the case $n \geq 5$ (equivalently, $\operatorname{dim} W_{\gamma} \geq 4$ ) curves in the Lagrangian Grassmannian of $W_{\gamma}$ have a nontrivial geometry with respect to the action of the linear symplectic group and any symplectic invariant of Jacobi curves of abnormal extremals produces an invariant of the original distribution $D$.

\section{Reduction to geometry of curves in projective spaces}

In the earlier works $[3,27]$ invariants of Jacobi curves were constructed using the notion of the cross-ratio of four points in Lagrangian Grassmannians analogous to the classical cross-ratio of four point in a projective line. Later, we developed a different method, leading to the construction of canonical bundles of moving frames and invariants for quite general curves in Grassmannians and flag varieties $[14,15]$. The geometry of Jacobi curves $J_{\gamma}$ in the case of rank 2 distributions can be reduced to the geometry of the so-called self-dual curves in the projective space $\mathbb{P} W_{\gamma}$.

For this first one can produce a curve of flags of isotropic/coisotropic subspaces of $W_{\gamma}$ by a series of osculations together with the operation of taking skew symmetric complements. For this, denote by $C\left(J_{\gamma}\right)$ the tautological bundle over $J_{\gamma}$ : the fiber of $C\left(J_{\gamma}\right)$ over the point $J_{\gamma}(\lambda)$ is the linear space $J_{\gamma}(\lambda)$. Let $\Gamma\left(J_{\gamma}\right)$ be the space of all smooth sections of $C\left(J_{\gamma}\right)$. If $\psi:(-\varepsilon, \varepsilon) \mapsto \gamma$ is a parametrization of $\gamma$ such that $\psi(0)=\lambda$, then for any $i \geq 0$ define

$$
\begin{gathered}
J_{\gamma}^{(i)}(\lambda):=\operatorname{span}\left\{\left.\frac{d^{j}}{d \tau^{j}} \ell(\psi(t))\right|_{t=0}: \ell \in \Gamma\left(J_{\gamma}\right), 0 \leq j \leq i\right\} \\
J_{\gamma}^{(-i)}(\lambda)=\left(J_{\gamma}^{(i)}(\lambda)\right)^{<}
\end{gathered}
$$

For $i>0$ we say that the space $J_{\gamma}^{(i)}(\lambda)$ is the $i$-th osculating space of the curve $J_{\gamma}$ at $\lambda$.

Note that $J_{\gamma}=J_{\gamma}^{(0)}$. Directly from the definitions the subspaces $J_{\gamma}^{(i)}(\lambda)$ are coisotropic for $i>0$ and isotropic for $i<0$ and the tuple $\left\{J_{\gamma}^{(i)}(\lambda)\right\}_{i \in \mathbb{Z}}$ defines a filtration of $W_{\gamma}$. In other words, the curve $\lambda \mapsto\left\{J_{\gamma}^{(i)}(\lambda)\right\}_{i \in \mathbb{Z}}$ is a curve of flags of $W_{\gamma}$. Besides, it can be shown [27] that

$$
\operatorname{dim} J^{(1)}(\lambda)-\operatorname{dim} J^{(0)}(\lambda)=\operatorname{dim} J^{(0)}(\lambda)-\operatorname{dim} J^{(-1)}(\lambda)=1,
$$

which in turn implies that $\operatorname{dim} J^{(i)}(\lambda)-\operatorname{dim} J^{(i-1)}(\lambda) \leq 1$, i.e. the jump of dimensions between the consecutive subspaces of the filtration $\left\{J_{\gamma}^{(i)}(\lambda)\right\}_{i \in \mathbb{Z}}$ is at most 1. This together with (3.6) implies that $\operatorname{dim} J_{\gamma}^{(i)}(\lambda) \leq n-3+i$ for $i>0$.

We say that $\lambda$ is a regular point of $\left(D^{2}\right)^{\perp} \backslash\left(D^{3}\right)^{\perp}$ if $\operatorname{dim} J_{\gamma}^{(i)}(\lambda)=n-3+i$ for $0<i \leq n-3$ or, equivalently, if $J_{\gamma}^{(n-3)}(\lambda)=W_{\gamma}$. A rank 2 distribution $D$ is called of maximal class at a point $q \in M$ if at least one point in $\pi^{-1}(q) \cap\left(D^{2}\right)^{\perp}$ is regular. Since by (2.4) the characteristic distribution $\mathscr{C}$ generated by a vector field depending algebraically on the fibers $\left(D^{2}\right)^{\perp}$, if $D$ is of maximal class at a point $q \in M$, then the set of all regular points of $\pi^{-1}(q) \cap\left(D^{2}\right)^{\perp}$ is non-empty open set in Zariski topology. The same argument is used to show that the set of germs of rank 2 distributions of maximal class is generic.

If $D$ is of maximal class at $q$ and $n \geq 5$, then by necessity $\operatorname{dim} D^{3}(q)=5$. The following question is still open: Does there exist a rank 2 distribution with $\operatorname{dim} D^{3}=5$ such that it is not of maximal 
class on some open set of $M$ ? We proved that the answer is negative for $n \leq 8$ and we have strong evidences that the answer is negative in general.

From now on we will work with rank 2 distributions of maximal class. In this case $\operatorname{dim} J_{\gamma}^{(4-n)}(\lambda)=1$, i.e. the curve $J_{\gamma}^{(4-n)}$ is a curve in the projective space $\mathbb{P} W_{\gamma}$. Moreover, the curve of flags $\lambda \mapsto\left\{J_{\gamma}^{(i)}(\lambda)\right\}_{i=3-n}^{n-3}, \lambda \in \gamma$ is the curve of complete flags and the space $J_{\gamma}^{(i)}(\lambda)$ is the $(i+n-4)$ th-osculating space of the curve $J_{\gamma}^{(4-n)}$. In other words, the whole curve of complete flags $\lambda \mapsto\left\{J_{\gamma}^{(i)}(\lambda)\right\}_{i=3-n}^{n-3}, \lambda \in \gamma$ can be recovered from the curve $J_{\gamma}^{(4-n)}$ and the differential geometry of Jacobi curves of abnormal extremals of rank 2 distributions is reduced to the differential geometry of curves in projective spaces.

\section{Canonical projective structure and Wilczynski invariants}

The differential geometry of curves in projective spaces is the classical subject, essentially completed already in 1905 by E.J. Wilczynski ( [25]). In particular, it is well known that these curves are endowed with the canonical projective structure, i.e., there is a distinguished set of parameterizations (called projective) such that the transition function from one such parametrization to another is a Möbius transformation. Let us demonstrate how to construct it for the curve $\lambda \mapsto J_{\gamma}^{(4-n)}(\lambda)$, $\lambda \in \gamma$.

As before, let $C\left(J_{\gamma}^{(4-n)}\right)$ be the tautological bundle $C\left(J_{\gamma}^{(4-n)}\right)$ over $J_{\gamma}^{(4-n)}$. Set $m=n-3$. Here we use a "naive approach", based on reparametrization rules for certain coefficient in the expansion of the derivative of order $2 m$ of certain sections of $C\left(J_{\gamma}^{(4-n)}\right)$ w.r.t. to the lower order derivatives of this sections. For the more algebraic point of view, based on Tanaka-like theory of curves of flags and $\mathfrak{s l}_{2}$-representations see $[9,14]$.

Take some parametrization $\psi: I \mapsto \gamma$ of $\gamma$, where $I$ is an interval in $\mathbb{R}$. By above, for any section $\ell$ of $C\left(J_{\gamma}^{(4-n)}\right)$ one has that

$$
\operatorname{span}\left\{\frac{d^{j}}{d t^{j}} \ell(\psi(t)) \mid 0 \leq j \leq 2 m-1\right\}=W_{\gamma} .
$$

A curves in the projective space $\mathbb{P} W_{\gamma}$ satisfying the last property is called regular (or convex). It is well known that there exists a unique, up to the multiplication by a nonzero constant, section $E$ of $C\left(J_{\gamma}^{(4-n)}\right)$, called a canonical section of $C\left(J_{\gamma}^{(4-n)}\right)$ with respect to the parametrization $\psi$, such that

$$
\frac{d^{2 m}}{d t^{2 m}} E(\psi(t))=\sum_{i=0}^{2 m-2} B_{i}(t) \frac{d^{i}}{d t^{i}} E(\psi(t)),
$$

i.e. the coefficient of the term $\frac{d^{2 m-1}}{d t^{2 m-1}} E(\psi(t))$ in the linear decomposition of $\frac{d^{2 m}}{d t^{2 m}} E(\psi(t))$ w.r.t. the basis $\left\{\frac{d^{i}}{d t^{i}} E(\psi(t)): 0 \leq i \leq 2 m-1\right\}$ vanishes.

Further, let $\psi_{1}$ be another parameter, $\widetilde{E}$ be a canonical section of $C\left(J_{\gamma}^{(4-n)}\right)$ with respect to the parametrization $\psi_{1}$, and $v=\psi^{-1} \circ \psi_{1}$. Then directly from the definition it easy to see that

$$
\widetilde{E}\left(\psi_{1}(\tau)\right)=c\left(v^{\prime}(\tau)\right)^{\frac{1}{2}-m} E(\psi(t))
$$

for some non-zero constant $c$.

Now let $\widetilde{B}_{i}(\tau)$ be the coefficient in the linear decomposition of $\frac{d^{2 m}}{d \tau^{2 m}} \widetilde{E}\left(\psi_{1}(\tau)\right)$ w.r.t. the basis $\left\{\frac{d^{i}}{d \tau^{i}} \widetilde{E}\left(\psi_{1}(t)\right): 0 \leq i \leq 2 m-1\right\}$ as in (5.2). Then, using the relation (5.3) it is not hard to show that 
the coefficients $B_{2 m-2}$ and $\widetilde{B}_{2 m-2}$ in the decomposition (5.2), corresponding to parameterizations $\psi$ and $\psi_{1}$, are related as follows:

$$
\widetilde{B}_{2 m-2}(\tau)=v^{\prime}(\tau)^{2} B_{2 m-2}(v(\tau))-\frac{m\left(4 m^{2}-1\right)}{3} \mathbb{S}(v)(\tau),
$$

where $\mathbb{S}(v)$ is the Schwarzian derivative of $v, \mathbb{S}(v)=\frac{d}{d \tau}\left(\frac{v^{\prime \prime}}{2 v^{\prime}}\right)-\left(\frac{v^{\prime \prime}}{2 v^{\prime}}\right)^{2}$.

From the last formula and the fact that $\mathbb{S} v \equiv 0$ if and only if the function $v$ is Möbius it follows that the set of all parameterizations $\varphi$ of $\gamma$ such that

$$
B_{2 m-2} \equiv 0
$$

defines the canonical projective structure on $\gamma$. Such parameterizations are called the projective parameterizations of the abnormal extremal $\gamma$. If $\psi$ and $\psi_{1}$ are two projective parametrizations, then there exists a Möbius transformation $v$ such that $\psi_{1}=\psi \circ v$.

Now let $t$ be a projective parameter on $J_{\gamma}^{(4-n)}$. E. Wilczynski showed that for any $i, 1 \leq i \leq$ $2 m-2$, the following degree $i+2$ differential

$$
\mathscr{W}_{i}(t) \stackrel{\text { def }}{=} \frac{(i+1) !}{(2 i+2) !}\left(\sum_{j=1}^{i}(-1)^{j-1} \frac{(2 i-j+3) !(2 m-i+j-3) !}{(i+2-j) ! j !} B_{2 m-3-i+j}^{(j-1)}(t)\right)(d t)^{i+2}
$$

on $J_{\gamma}^{(4-n)}$ does not depend on the choice of the projective parameter. In other words, for any $\lambda \in \gamma$, $\mathscr{W}_{i}$ is the well defined homogeneous polynomial of degree $i+2$ on the tangent line to $J_{\gamma}(\lambda)$ or, equivalently, on the tangent line to the abnormal extremal $\gamma$ at $\lambda$. The form $\mathscr{W}_{i}$ is called the $(i+2)$ nd order Wilczynski invariant of the curve $J_{\gamma}^{(4-n)}$.

In a given parametrization of $\gamma$ one can compute the Wilczynski invariant $\mathscr{W}_{i}$ on the velocity vectors (corresponding to this parametrization) at any points of the curve $\gamma$ to obtain a function on the curve. This function (which is sometimes also referred as the density of the Wilczynski invariant in the given parameter or simply as the Wilczynski invariant itself) is a relative invariant, because in another parameter it is multiplied by the derivative of the reparametrization (from the new parameter to the old one) raised to $(i+2)$-nd power (equal to the degree of the Wilczynski invariant).

Remark 5.1. Among all regular curves in the projective space $\mathbb{P}^{k}$ of dimension $k$, the curves with all Wilczynski invariants equal to zero belong to the rational normal curve, i.e. to the curve consisting of the points of the form $\left[t^{k}: t^{k-1} s \ldots: t s^{k-1}: s^{k}\right]$ in some homogeneous coordinates.

Note that the curve $J_{\gamma}^{(4-n)}$ is not an arbitrary regular curve in the projective space $\mathbb{P} W$. It satisfies the following additional property:

(S1) The $(n-4)$ th-osculating space of $J_{\gamma}^{(4-n)}$ at any point $\lambda$ is Lagrangian.

As shown already by Wilczynski [25] such curves are self-dual in the following sense:

(S2) The curve $\left(J_{\gamma}^{(n-4)}\right)^{*}$ in the projectivization $\mathbb{P} W_{\gamma}^{*}$ of the dual space $W_{\gamma}^{*}$, which is dual to the curve of hyperplanes $J_{\gamma}^{(n-4)}$ obtained from the original curve $J_{\gamma}^{(4-n)}$ by the osculation of order $2(n-4)$, is equivalent to the original curve $J_{\gamma}^{(4-n)}$, i.e. there is a linear transformation $A: W \mapsto W^{*}$ sending $J_{\gamma}^{(n-4)}$ onto $\left(J_{\gamma}^{(n-4)}\right)^{*}$.

Note that in contrast to property (S1) the formulation of property (S2) does not involve a symplectic structure on $W_{\gamma}$. However, it can be shown $[20,25]$ that if the property (S2) holds then there 
exists a unique, up to a multiplication by a nonzero constant, symplectic structure on $W_{\gamma}$ such that the property (S1) holds (here it is important that $\operatorname{dim} W_{\gamma}$ is even; similar statement for the case of odd dimensional linear space involves nondegenerate symmetric forms instead of skew-symmetric ones). Since in our case the symplectic structure on $W_{\gamma}$ is a priori given, in the sequel we will consider projective spaces of linear symplectic spaces only and by self-dual curves we will mean curves satisfying property $(\mathrm{S} 1)$.

It was shown by Wilczynski that a curve in a projective space is self-dual if an only if all Wilczynski invariant of odd order vanish (for the modern Lie-algebraic interpretation of this fact see [14]). The remaining $n-4$ Wilczynski invariants of even order, $\mathscr{W}_{2 i}, 1 \leq i \leq n-4$, constitute the fundamental set of symplectic invariants of the unparametrized curve $J_{\gamma}^{(4-n)}$. Note that the nonzero Wilczynski invariants have order $\geq 4$ in this case and $\mathscr{W}_{2}=B_{2 m-4}(t)(d t)^{4}$ in any projective parameter $t$.

Taking the Wilczynski invariants $\mathscr{W}_{2 i}$ for the Jacobi curves of all abnormal extremals living in the set $\mathscr{R}_{D}$ we obtain the invariants of the distribution $D$, called the generalized Wilczynski invariants of $D$ of order $2(i+1)$ and denoted also by $\mathscr{W}_{2 i}$. By the constructions, the generalized Wilczynski invariant $\mathscr{W}_{2 i}$ at a regular point $\lambda$ of $\left(D^{2}\right)^{\perp} \backslash\left(D^{3}\right)^{\perp}$, is a special homogeneous polynomial of degree $2(i+1)$ on the tangent line at $\lambda$ to the abnormal extremal passing through $\lambda$. Another interpretation of these invariants, as certain functions on fibers of $\left(D^{2}\right)^{\perp}$, defined by a multiplication on a constant on a fiber, is given in [27].

Note that in the case $n=5$ the only possibly nonzero generalized Wilczynski invariant is $\mathscr{W}_{2}$ and it has degree 4. As shown in [28], under an appropriate identification, this invariant coincides with the classical Cartan invariant obtained in [8].

\section{Canonical frames for rank 2 distributions of maximal class}

Now let $\mathscr{R}_{D}$ be the set if all regular points of $\left(D^{2}\right)^{\perp} \backslash\left(D^{3}\right)^{\perp}$. Denote by $\mathfrak{P}_{\lambda}$ the set of all projective parameterizations $\psi$ on the characteristic curve $\gamma$, passing through $\lambda$, such that $\psi(0)=\lambda$. Let

$$
\Sigma_{D}=\left\{(\lambda, \psi): \lambda \in \mathscr{R}_{D}, \psi \in \mathfrak{P}_{\lambda}\right\}
$$

Actually, $\Sigma_{D}$ is a principal bundle over $\mathscr{R}_{D}$ with the structural group of all Möbius transformations, preserving 0 and $\operatorname{dim} \Sigma_{D}=2 n-1$. The main results of $[10,11]$ can be summarized in the following:

Theorem 6.1. For any rank 2 distribution in $\mathbb{R}^{n}$ with $n>5$ of maximal class there exists the canonical, up to the action of $\mathbb{Z}_{2}$, frame on the corresponding $(2 n-1)$-dimensional manifold $\Sigma_{D}$ so that two distributions from the considered class are equivalent if and only if their canonical frames are equivalent.

The group of symmetries of such distributions is at most $(2 n-1)$-dimensional and this upper bound is sharp. All distributions from the considered class with $(2 n-1)$-dimensional Lie algebra of infinitesimal symmetries is locally equivalent to the distribution $D_{((0, \ldots 0)}$ generated by the vector fields $X_{1}$ and $X_{2}$ from (1.2)-(1.3) with all $r_{i}$ equal to 0 or, equivalently, associated with the underdetermined $O D E z^{\prime}(x)=\left(y^{(n-3)}(x)\right)^{2}$.

The symmetry algebra of this distribution is isomorphic to a semidirect sum of $\mathfrak{g l}(2, \mathbb{R})$ and $(2 n-5)$-dimensional Heisenberg algebra $\mathfrak{n}_{2 n-5}$ such that $\mathfrak{g l}(2, \mathbb{R})$ acts irreducibly on a complement of the center of $\mathfrak{n}_{2 n-5}$ to $\mathfrak{n}_{2 n-5}$ itself. 


\section{Canonical frames for rank 2 distributions of maximal class with distinguish parametrization on abnormal extremals}

Now assume that at least one generalized Wilczynski invariant of rank 2 distribution $D$ of maximal class does not vanish. Let $i_{0}$ be the minimal integer such that $\mathscr{W}_{2 i_{0}} \neq 0$ on some (open) subset $\widetilde{\mathscr{R}}$ of $\mathscr{R}_{D}$. Then on each segment $\gamma$ of abnormal extremals lying in $U$ we can choose the unique, up to a shift and the change of the orientation, parametrization $\psi: I \mapsto \gamma$ such that

$$
\mathscr{W}_{2 i_{0}}(\psi(t))\left(\psi^{\prime}(t)\right) \equiv \varepsilon,
$$

where $\varepsilon=1$ if $\mathscr{W}_{2 i_{0}}>0$ and $\varepsilon=-1$ if $\mathscr{W}_{2 i_{0}}<0$. Here by $\mathscr{W}_{2 i_{0}}(\lambda)(v)$, where $\lambda \in U$ and $v$ is the tangent vector at $\lambda$ to the abnormal extremal $\gamma$ passing to $\lambda$, we mean the generalized Wilczynski invariant $\mathscr{W}_{2 i_{0}}$ at $\lambda$ evaluated at $v$.

Moreover, we also can fix the orientation on the curve. For this note that since the curve $J_{\gamma}^{(4-n)}$ is self-dual, given a parametrization $\psi$ on $\gamma$, among all canonical sections of the tautological bundle $C\left(J_{\gamma}^{(4-n)}\right)$ (defined up to the multiplication by a nonzero constant) there exists the unique, up to a sign, section $E$ of such that (5.2) holds and

$$
\left|\tilde{\sigma}_{\gamma}\left(\frac{d^{n-3}}{d t^{n-3}} E(\psi(t)), \frac{d^{n-4}}{d t^{n-4}} E(\psi(t))\right)\right| \equiv 1 .
$$

This section $E$ will be called the strongly canonical section of $C\left(J_{\gamma}^{(4-n)}\right)$ with respect to the parametrization $\psi$. The parametrization $\psi$ is called the canonical parametrization of the abnormal extremal $\gamma$ if (7.1) holds and

$$
\tilde{\sigma}_{\gamma}\left(\frac{d^{n-3}}{d t^{n-3}} E(\psi(t)), \frac{d^{n-4}}{d t^{n-4}} E(\psi(t))\right) \equiv 1 .
$$

Note that the canonical parametrization is preserved by the homotheties of the fibers of $\left(D^{2}\right)^{\perp}$. Namely, if $\delta_{s}$ is the flow of homotheties on the fibers of $T^{*} M: \delta_{s}(p, q)=\left(e^{s} p, q\right), \quad q \in M, p \in$ $T_{q}^{*} M$ or, equivalently, the flow generated by the Euler field $e$ generates this flow, then $\psi: I \mapsto \gamma$ is the canonical parametrization on an abnormal extremal $\gamma$ if and only if $\delta_{s} \circ \psi$ is the canonical parametrization on the abnormal extremal $\delta_{s} \circ \gamma$.

The main goal of this section is to prove the following

Theorem 7.1. Given a rank 2 distribution $D$ of maximal class with at least one nonvanishing generalized Wilczynski invariant one can assign to such structure a canonical, up to the action of $\mathbb{Z}_{2}$, frame on the set $\widetilde{\mathscr{R}}$ defined above so that two objects from the considered class are equivalent if and only if their canonical frames are equivalent.

Proof. Given $\lambda \in\left(D^{2}\right)^{\perp}$ denote by $V(\lambda)$ the tangent space to the fiber of the bundle $\pi:\left(D^{2}\right)^{\perp} \mapsto M$ (the vertical subspace of $T_{\lambda}\left(D^{2}\right)^{\perp}$ ),

$$
V(\lambda)=\left\{v \in T_{\lambda}\left(D^{2}\right)^{\perp}, \pi_{*} v=0\right\} .
$$

It is easy to show ( $[11,27])$ that

$$
d \phi(V(\lambda) \oplus \mathscr{C}(\lambda))=J_{\gamma}^{(-1)}(\lambda) \bmod \mathbb{R} \bar{e}
$$

where $\phi$ is as in (3.2), $\bar{e}=\phi_{*} e$ with $e$ being the Euler field, and $\gamma$ is the abnormal extremal passing through $\lambda$. Define also the following subspaces of $T_{\lambda}\left(D^{2}\right)^{\perp}$ : 


$$
\mathscr{J}^{(i)}(\lambda)=\left\{w \in T_{\lambda}\left(D^{2}\right)^{\perp}: d \phi(w) \in J_{\gamma}^{(i)}(\lambda) \bmod \mathbb{R} \bar{e}\right\}
$$

Directly from the definition, if $\lambda \in \mathscr{R}_{D}$, then

$$
\left[\mathscr{C}, \mathscr{J}^{(i)}\right](\lambda)=\mathscr{J}^{(i+1)}(\lambda)
$$

Also, if $V^{(i)}(\lambda)=V(\lambda) \cap \mathscr{J}^{(i)}(\lambda)$, then

$$
\mathscr{J}^{(i)}(\lambda)=V^{(i)}(\lambda) \oplus \mathscr{C}(\lambda) \quad \forall i<0
$$

Moreover, it can be shown ( [11, Lemma 2]) that

$$
\left[V^{(i)}, V^{(i)}\right] \subseteq V^{(i)}, \quad\left[V^{(i)}, \mathscr{J}^{(-i)}\right] \subseteq \mathscr{J}^{(-i)}, \quad \forall i \leq 0
$$

Let $E$ be the strongly canonical section of $C\left(J_{\gamma}^{(4-n)}\right)$ with respect to the canonical parametrization $\psi$ of the abnormal extremal $\gamma$ (as defined by (7.2)). Then (7.5) implies that a vector field $\varepsilon_{1}$ such that

(A1) $d \phi\left(\varepsilon_{1}(\lambda)\right) \equiv E \bmod \bar{e}$

(A2) $\varepsilon_{1}$ is the section of the vertical distribution $V$

is defined modulo the Euler field $e$. Note that conditions (A1) and (A2) also imply that $\varepsilon_{1}$ is the section of $V^{(4-n)}$.

Now let $h$ be the vector field consisting of the tangent vectors to the abnormal extremals parameterized by the canonical parameter. Note that $h$ is a section of the characteristic line distribution $\mathscr{C}$.

Lemma 7.1. Among all vector fields $\varepsilon_{1}$ satisfying conditions (A1) and (A2), there exists the unique, up to a multiplication by -1 , vector field such that

$$
\left[\varepsilon_{1},\left[h, \varepsilon_{1}\right]\right](\lambda) \in \operatorname{span}\left\{e(\lambda), h(\lambda), \varepsilon_{1}(\lambda)\right\}
$$

Proof. Let $\tilde{\varepsilon}_{1}$ be a vector field satisfying the conditions (A1) and (A2). Then $\tilde{\varepsilon}_{1}$ is the section of $V^{(4-n)}$. From (7.7) it follows that $\left[h, \tilde{\varepsilon}_{1}\right]$ is a section of $\mathscr{J}^{(5-n)}$. If $n>5$ this together with the first relation in (7.8) implies that $\left[\tilde{\varepsilon}_{1},\left[h, \tilde{\varepsilon}_{1}\right]\right]$ is a section of $\mathscr{J}^{(5-n)}$. For $n=5$ the same is true because of the second relation in (7.8) applied for $i=0$. Note that again by (7.7) we have

$$
\mathscr{J}^{(5-n)}=\operatorname{span}\left\{e, h, \tilde{\varepsilon}_{1},\left[h, \tilde{\varepsilon}_{1}\right]\right\}
$$

Therefore, 


$$
\left[\tilde{\varepsilon}_{1},\left[h, \tilde{\varepsilon}_{1}\right]\right] \equiv k\left[h, \tilde{\varepsilon}_{1}\right] \bmod \operatorname{span}\left\{\mathrm{e}, \mathrm{h}, \tilde{\varepsilon}_{1}\right\}
$$

for some function $k$. Now let $\varepsilon_{1}$ be another vector field satisfying conditions (A1) and (A2). Then by above there exists a function $\mu$ such that

$$
\varepsilon_{1}= \pm \tilde{\varepsilon}_{1}+\mu e .
$$

From the fact that the canonical parametrization is preserved by the homotheties of the fibers of $\left(D^{2}\right)^{\perp}$ it follows that $[e, h]=0$. Also from the normalization condition (7.2) it is easy to get that

$$
\left[e, \varepsilon_{1}\right]=-\frac{1}{2} \varepsilon_{1} \bmod \operatorname{span}(e)
$$

Then

$$
\left[e,\left[h, \varepsilon_{1}\right]\right]=-\frac{1}{2}\left[h, \varepsilon_{1}\right] \bmod (e, h),
$$

From this and (7.12) it follows that

$$
\left[\varepsilon_{1},\left[h, \varepsilon_{1}\right]\right] \equiv\left(k \mp \frac{\mu}{2}\right)\left[h, \varepsilon_{1}\right] \operatorname{span}\left\{e, h, \varepsilon_{1}\right\},
$$

which implies the statement of the lemma: the required vector $\tilde{\varepsilon}_{1}$ is obtained by taking $\mu= \pm 2 k$.

Now we are ready to construct the canonical frame on the set $\widetilde{\mathscr{R}}$. One option is to take as a canonical frame the following one:

$$
\left\{e, h, \varepsilon_{1},\left\{(\operatorname{ad} h)^{i} \varepsilon_{1}\right\}_{i=1}^{2 n-7},\left[\varepsilon_{1},(\operatorname{ad} h)^{2 n-7} \varepsilon_{1}\right]\right\},
$$

where $\varepsilon_{1}$ is as in Lemma 7.1. Let us explain why it is indeed a frame. First the vector fields $\left\{e, h, \varepsilon_{1},\left\{(\operatorname{ad} h)^{i} \varepsilon_{1}\right\}_{i=1}^{2 n-7}\right\}$ are linearly independent on $\widetilde{\mathscr{R}}$ due to the relation (7.7). Besides,

$$
\left[\varepsilon_{1},(\operatorname{ad} h)^{2 n-7} \varepsilon_{1}\right](\lambda) \notin \mathscr{J}^{(n-3)}(\lambda) .
$$

Otherwise, $\varepsilon_{1}(\lambda)$ belongs to the kernel of the form $\left.\sigma(\lambda)\right|_{\left(D^{2}\right)^{\perp}}$ and therefore it must be collinear to $h$. We get a contradiction. Therefore the tuple of vectors in (7.16) constitute a frame on $\widetilde{\mathscr{R}}$.

The construction of the frame (7.16) is intrinsic. However, in order to guaranty that two objects from the considered class are equivalent if and only if their canonical frames are equivalent, we have to modify this frame such that it will contain the basis of the vertical distribution $V$ (defined by 7.4). For this, replace the vector fields of the form $(\operatorname{ad} h)^{i} \varepsilon_{1}$ for $1 \leq i \leq n-4$ by their projections to $V^{(i)}$ with respect to the splitting (7.8), i.e. their vertical components with respect to this splitting. This completes the construction of the required canonical frame (defined up to the action of the required finite groups). The proof of Theorem 7.1 is completed.

As a direct consequence of Theorem 7.1 we have

Corollary 7.1. For a rank 2 distribution $D$ of maximal class with at least one nonvanishing generalized Wilczynski invariant the dimension of pseudo-group of local symmetries does not exceed $2 n-3$. 


\section{Symplectic curvatures for the structures under consideration}

Before proving Theorems 1.1 about the most symmetric models for geometric structures under consideration, we want to reformulate this theorem in more geometric terms. For this we distinguish special invariants for this structures, called the symplectic curvatures. In contrast to the generalized Wilczynski invariants they are functions on the open subset $\widetilde{R}$ of $\mathscr{R}_{D}$, defined in the beginning of the previous section.

First assume that $J_{\gamma}^{(n-4)}$ is parametrized by the parametrization $\psi$ which is not necessary canonical. The geometry of parameterized regular self-dual curves in projective spaces is simpler than of unparametrized ones: instead of forms on the curve we obtain invariant, which are scalar-valued function on the curve ( [29]). The main result of [29] (Theorem 2 there) can be reformulated as follows (see also [20]: if $E$ is a (strongly) canonical section of $C\left(J_{\gamma}^{(4-n)}\right)$ with respect to the (canonical) parametrization $\psi$, then there exist $m$ functions $\rho_{1}(t), \ldots, \rho_{m}(t)$ such that

$$
E^{(2 m)}(\psi(t))=\sum_{i=1}^{m}(-1)^{i+1} \frac{d^{m-i}}{d t^{m-i}}\left(\rho_{i}(t) \frac{d^{m-i}}{d t^{m-i}} E(\psi(t))\right) .
$$

Note that formula (8.1) resembles the classical normal form for the formally self-adjoint linear differential operators [21][§1].

By constructions, the functions $\rho_{1}(t), \ldots, \rho_{m}(t)$ are invariants of the parameterized curve $t \mapsto$ $J_{\gamma}^{(4-n)}(\psi(t))$ with respect to the action of the linear symplectic group on $W_{\gamma}$. We call the function $\rho_{i}(t)$ the ith symplectic curvature of the parametrized curve $t \mapsto J_{\gamma}^{(4-n)}(\psi(t))$. Besides, the functions $\rho_{1}(t), \ldots, \rho_{m}(t)$ constitute the fundamental system of symplectic invariant of the parametrized curve $t \mapsto J_{\gamma}^{(4-n)}(\psi(t))$, i.e. they determine this curve uniquely up to a symplectic transformation. Moreover, these invariants are independent: for any tuple of $m$ functions $\rho_{1}(t), \ldots, \rho_{m}(t)$ on the interval $I \subseteq R$ there exists a parameterized regular self-dual curve $t \mapsto \Lambda(t), t \in I$, in the projective space of dimension $2 m-1$ with the $i$ th symplectic curvature equal to $\rho_{i}(t)$ for any $1 \leq i \leq m$.

Also in the sequel we will need the following

Remark 8.1. Assume that $E$ is the strongly canonical section of $C\left(J_{\gamma}^{(4-n)}\right)$ with respect to the parametrization $\psi$. Using the fact that the spaces $\operatorname{span}\left\{\frac{d^{j}}{d t^{j}} E(\psi(t))\right\}_{j=1}^{m}$ are Lagrangian and the condition (7.2), it is easy to show that

$$
\tilde{\sigma}_{\gamma}\left(\frac{d^{j}}{d t^{j}} E(\psi(t)), \frac{d^{i}}{d t^{i}} E(\psi(t))\right)
$$

are either identically equal to 0 , if $i+j<2 m-1$ or to \pm 1 , if $i+j=2 m-1$, or they are polynomial expressions (with universal constant coefficients) with respect to the symplectic curvatures $\rho_{1}(t), \ldots, \rho_{m}(t)$ and their derivatives, if $i+j>2 m$.

Assume that at least one generalized Wilczynski invariant of rank 2 distribution $D$ of maximal class does not vanish identically. Let $i_{0}$ be the minimal integer such that $\mathscr{W}_{2 i_{0}}$ on some (open) subset $\widetilde{\mathscr{R}}$ of $\mathscr{R}_{D}$. Now let $\psi$ be the canonical, up to a shift, parametrization of an abnormal extremal $\gamma$ given by (7.1) and (7.3). Let $\rho_{i}$ be the $i$ th symplectic curvatures of the parametrized curve $t \mapsto$ $J_{\gamma}^{(4-n)}(\psi(t))$. Then $\rho_{i}$ is an invariant of the unparametrized curve $J_{\gamma}^{(4-n)}$ with respect to the action of the linear symplectic group on $W_{\gamma}$ and a reparametrization of $\gamma$, because the parametrization $\psi$ 
on $\gamma$ is determined by the unparametrized curve $J_{\gamma}^{(4-n)}$ itself. We say that $\rho_{i}$ is the ith symplectic curvature of the unparametrized curve $J_{\gamma}^{(4-n)}$.

Remark 8.2. Note that by 5.4 the first symplectic curvature $\rho_{1}$ is equal, up to the universal constant multiple $\frac{-m\left(4 m^{2}-1\right)}{3}$, to the Schwarzian derivative of the transition function from the canonical parametrization of $\gamma$ to any projective parametrization of $\gamma$. The invariant $\rho_{1}$ coincides here, up to the universal constant, with the projective Ricci curvature of the unparametrized curve in the Lagrangian Grassmannian introduced in $[27,31]$.

In contrast to the symplectic curvatures of a parametrized curves, the symplectic curvatures of an unparametrized curve are dependent so that there are $i_{0}$ polynomial relations (with universal coefficients) between them and their derivatives (with respect to the canonical parametrization). Therefore the following definition makes sense.

Definition 8.1. The tuple of functions $\left(r_{1}(\tau), \ldots, r_{m}(\tau)\right)$ is called compatible if there exists a regular self-dual curve $\Lambda$ in the projective space of dimension $2 m-1$ with at least one non-vanishing Wilczynski invariant such that if $\rho_{i}$ is the $i$ th symplectic curvature of the unparametrized curve $\Lambda$, then for all $1 \leq i \leq m$ we have that $r_{i}(\tau)=\rho_{i}(\psi(\tau))$ for the canonical parametrization $\psi$ of $\Lambda$.

To explain why the invariants $\left(r_{1}(\tau), \ldots, r_{m}(\tau)\right)$ are dependent note that there is another way to construct the scalar-valued invariant of the unparametrized curve $J_{\gamma}^{(4-n)}$ (up to a symplectic transformation and reparametrization) with the help of the Wilczynski invariants. Namely, for any $1 \leq i \leq m-1$ let

$$
A_{i}(\psi(t))=\mathscr{W}_{2 i}(\psi(t))\left(\psi^{\prime}(t)\right)
$$

Then by the definition of $i_{0}$ and the canonical parametrization, $A_{i} \equiv 0$ for $1 \leq i \leq i_{0}-1$ and $A_{i_{0}}=\varepsilon$, where $\varepsilon=1$ if $\mathscr{W}_{2 i_{0}}>0$ and $\varepsilon=-1$ if $\mathscr{W}_{2 i_{0}}<0$. Using the transformation rule (5.3) with $\psi$ being a projective parametrization of $\gamma$ and $\psi_{1}$ being the canonical parametrization of $\gamma$ and also the Remark 8.2 , it can be shown that all $\rho_{i}$ with $1 \leq i \leq i_{0}$ can be expressed as a certain polynomial in $\rho_{1}$ and its derivatives (with respect to the canonical parameter) having universal coefficients and with a free term equal to zero if $1 \leq i<i_{0}$ and equal to $(-1)^{i_{0}-1} \varepsilon$ if $i=i_{0}$. For example, if $i_{0}=1$, i.e. the nontrivial Wilczynski invariant $W_{2}$ of the lowest order is non-zero, then there is only one relation

$$
\rho_{2}\left(\psi_{1}(\tau)\right)=-\varepsilon-\frac{3(2 m-2)(2 m-3)}{20} \frac{d^{2}}{d \tau^{2}} \rho_{1}(\psi(\tau))+\frac{\alpha_{m, 2}}{\alpha_{m, 1}^{2}} \rho_{1}(\psi(\tau))^{2},
$$

where the constants $\alpha_{m, 1}$ and $\alpha_{m, 2}$ are given by formula 1.5. Therefore a tuple $\left(r_{1}(\tau), \ldots, r_{m}(\tau)\right)$ satisfying relation (8.3), with $\rho_{i}(\psi(\tau))$ is replaced by $r_{i}(\tau)$, is compatible. Relation (8.3) is analogous to [3, Lemma 5.1]. The first relation for $i_{0}>1$ is obtained from (8.3) by replacing $\varepsilon$ with 0 .

The deduction of other relations in an explicit form in general needs an extra work and we will not do it here, because we do not need such explicit relations in the sequel. However, in the case when all invariants $\rho_{i}(\tau)$ are constants, $\rho_{i}(t) \equiv r_{i}, 1 \leq i \leq m$, there is much more elegant way to explain the role of the coefficients of polynomial (1.5) in the question of compatibility of the tuple $\left(r_{1}, \ldots, r_{m}\right)$, based on some elementary facts from the representation theory of the Lie algebra $\mathfrak{s l}_{2}$. 
Given a tuple of $m$ numbers $\left(r_{1}, \ldots, r_{m}\right)$ let $\tau \mapsto \Lambda_{\left(r_{1}, \ldots, r_{m}\right)}(\tau), \tau \in R$ be the parameterized selfdual curve in the $(2 m-1)$-dimensional projective space $\mathbb{P}^{2 m-1}$ with the $i$ th symplectic curvature constantly equal to $r_{i}$ for all $1 \leq i \leq m$. Note that the closure of the curve $\Lambda_{(0, \ldots, 0)}$ is the rational normal curve.

Proposition 8.1. The curve $\Lambda_{\left(r_{1}, \ldots, r_{m}\right)}$ has all Wilczynski invariants equal to zero if and only if the tuple $\left(r_{1}, \ldots, r_{m}\right)$ is exceptional in the sense of Definition 1.1.

Proof. Assume that the curve $\Lambda_{\left(r_{1}, \ldots, r_{m}\right)}$ has all Wilczynski invariants equal to zero. Then by Remark 5.1 $\Lambda_{\left(r_{1}, \ldots, r_{m}\right)}$ belongs to a rational normal curve. It is well known $[9,15,25]$ that the algebra of infinitesimal symmetries of the rational normal curve (with respect to the action of $S L_{2 m}$ ) is isomorphic to $\mathfrak{s l}_{2}$ and it is actually equal to the image of the irreducible embedding of $\mathfrak{s l}_{2}$ into $\mathfrak{s l}_{2 m}$.

To describe these infinitesimal symmetries note that $\mathbb{R}^{2 m}$ can be identified with the projectivization of the space of homogeneous binary polynomials of degree $2 m-1$ (say in variables $x_{1}$ and $x_{2}$ ), $\mathbb{R}^{2 m} \cong \operatorname{Sym}^{2 m-1}\left(\mathbb{R}^{2}\right)$. With this identification, the standard action of the group $S L_{2}$ (the algebra $\mathfrak{s l}_{2}$ ) on $\mathbb{R}^{2}$ with coordinates $\left(x_{1}, x_{2}\right)$ induces the standard irreducible representation of $\left.S L_{2}(\mathfrak{s l})_{2}\right)$ into $S L_{2 m}$ $\left(\mathfrak{s} l_{2 m}\right)$ The rational normal curve $\bar{\Lambda}_{(0, \ldots, 0)}$, up to a projective transformation, is the projectivization of the binary polynomials which are the $(2 m-1)$ th power of the linear forms in $x_{1}$ and $x_{2}$. Therefore any element of the image of the standard irreducible representation of $S L_{2}$ in $S L_{2 m}$ preserves the curve $\bar{\Lambda}_{(0, \ldots, 0)}$ and any element of the image of the standard irreducible representation of $\mathfrak{s l}_{2}$ in $\mathfrak{s l}_{2 m}$ defines an infinitesimal symmetry of this curve. Moreover, it can be shown that there are no other infinitesimal symmetries of this curve.

By the standard theory of $\mathfrak{s l}_{2}$-representations $([17, \S 11.1])$ the image under this representation of any diagonizable element of $\mathfrak{s l}_{2}$ has the spectrum of the form

$$
\{-(2 m-1) r,-(2 m-3) r, \ldots,-r, r, \ldots,(2 m-3) r,(2 m-1) r\} .
$$

(where over $\mathbb{R}$ the number $r$ is either real or purely imaginary), which is an arithmetic progression symmetric with respect to the origin. Besides any element of $\mathfrak{s l}_{2}$ can be brought to the triangular form (over $\mathbb{C}$ ), therefore its image under the aforementioned embedding has also the spectrum of the form (8.4). In other words, the spectrum of any infinitesimal symmetry of $\Lambda_{\left(r_{1}, \ldots, r_{m}\right)}$ is an arithmetic progression symmetric with respect to the origin.

On the other hand, since all symplectic invariants of the curve $\tau \mapsto \Lambda_{\left(r_{1}, \ldots, r_{m}\right)}(\tau)$ are constants, this curve belongs to the orbit of the one-parametric group generated by an element $X_{\left(r_{1}, \ldots, r_{m}\right)}$ of the symplectic algebra (for the explicit form of $X_{r_{1}, \ldots, r_{m}}$ see [29], where it is exactly the matrix in the structure equation for the canonical moving frame of the curve $\left.\tau \mapsto \Lambda_{\left(r_{1}, \ldots, r_{m}\right)}(\tau)\right)$. Therefore $X_{\left(r_{1}, \ldots, r_{m}\right)}$ belongs to the algebra of infinitesimal symmetries of the curve $\Lambda_{\left(r_{1}, \ldots, r_{m}\right)}$. Hence, by above its spectrum is an arithmetic progression symmetric with respect to the origin. Finally, from the explicit form of $X_{\left(r_{1}, \ldots, r_{m}\right)}$ given in [29] it follows that the characteristic polynomial of $X_{\left(r_{1}, \ldots, r_{m}\right)}$ is exactly the polynomial (1.4), which completes the proof of one direction of the proposition.

In opposite direction, assume that the tuple $\left(r_{1}, \ldots, r_{m}\right)$ is exceptional in the sense of Definition 1.1. Then the corresponding element $X_{\left(r_{1}, \ldots, r_{m}\right)}$ has the matrix $S+N$ in some basis, where $S$ is the diagonal matrix with the entries on the diagonal as in (8.4) for some $r$ (and in the same order) and $N$ is the Jordan block. Then from the assumptions on the spectrum of $X_{\left(r_{1}, \ldots, r_{m}\right)}$ it follows that in this basis $X_{\left(r_{1}, \ldots, r_{m}\right)}$ can be considered as an element of the image of the standard irreducible embedding of $\mathfrak{s l}_{2}$ into $\mathfrak{s l}_{2 m}$. This embedding is the algebra of infinitesimal symmetries of the orbit of the first coordinate line with respect to the one-parametric group generated by $N$. Consequently, our curve 
$\Lambda_{\left(r_{1}, \ldots, r_{m}\right)}$ belongs to the closure of this orbit, which in turn is a rational normal curve. This completes the proof of our proposition.

From Proposition 8.1 it follows that an exceptional tuple $\left(r_{1}, \ldots, r_{m}\right)$ in the sense of Definition 1.1 is not compatible. Another consequence is the following

Corollary 8.1. The distribution $D_{\left(r_{1}, \ldots, r_{n-3}\right)}$ is locally equivalent to $D_{(0, \ldots, 0)}$ (or, equivalently, has the algebra of infinitesimal symmetries of the maximal possible dimension among all rank 2 distributions of maximal class in $\left.\mathbb{R}^{n}\right)$ if and only if the tuple $\left(r_{1}, \ldots, r_{n-3}\right)$ is exceptional in the sense of Definition 1.1.

Proof. In [12] we established that the following three equivalence problems are the same after an appropriate identification of the objects involved in them: the equivalence of rank 2 distributions of a special type, namely, associated with underdetermined ODE (the Monge equation) $z^{\prime}(x)=F\left(x, y(x), \ldots, y^{(n-3)}(x)\right)$, the equivalence of the Lagrangians as in (1.7), up to a contact transformation, a multiplication by a nonzero constant, and modulo divergence, and the equivalence of their Euler-Lagrange equations, up to a contact transformation. Moreover, the latter problem, in the case when the Euler-Lagrangian equation is linear coincides with the equivalence of the corresponding self-dual curves in projective spaces (up to a linear symplectic transformation). The distribution $D_{\left(r_{1}, \ldots, r_{n-3}\right)}$ is associated with the Monge equation (1.1), which in turn corresponds to the Lagrangian (1.7) having the linear Euler-Lagrange equation. So, the question of equivalence of the distributions $D_{\left(r_{1}, \ldots, r_{n-3}\right)}$ and $D_{(0, \ldots, 0)}$ is reduced to the question of the equivalence of the curves $\Lambda_{\left(r_{1}, \ldots, r_{m}\right)}$ and $\Lambda_{(0, \ldots, 0)}$ from Proposition 8.1. Hence, our Corollary follows from Proposition 8.1.

The following simple lemma will be useful in the next section

Lemma 8.1. Let $\left(r_{1}, r_{2} \ldots, r_{m}\right)$ be a tuple of $m$ constants which is not exceptional in the sense of Definition 1.1. Then among all tuple of the form $\left(c^{2} r_{1}, c^{4} r_{2}, \ldots, c^{2 m} r_{m}\right)$, where $c$ is an arbitrary non-zero constant, there exists exactly one tuple which is compatible in the sense of Definition 8.1

Proof. Let the curve $\tau \mapsto \Lambda_{\left(r_{1}, r_{2} \ldots, r_{m}\right)}(\tau)$ be as in Proposition 8.1. Then since all $r_{i}$ are constants, the germs of the curve $\Lambda$ at two different time moments $\tau_{1}$ and $\tau_{2}$ are equivalent, up to a symplectic transformation, i.e there exist a symplectic transformation $S$ that sends the germ of $\Lambda_{\left(r_{1}, r_{2} \ldots, r_{m}\right)}$ at $\tau_{1}$ to the germ of this curve at $\tau_{2}$. In particular, if $E(\tau)$ is the germ of the canonical sections of the parametrized curve $\tau \mapsto \Lambda_{\left(r_{1}, r_{2} \ldots, r_{m}\right)}(\tau)$ at $\tau=\tau_{1}$, then $S E\left(\tau+\tau_{1}-\tau_{2}\right)$ is the germ of the canonical section of this curve at $\tau=\tau_{2}$. Therefore, all coefficients $B_{i}$ in the expansion (5.2) are constants and consequently the Wilczynski invariants have the form $\mathscr{W}_{2 i}=A_{i}(d \tau)^{2 i+2}, 1 \leq i \leq m-1$, where all $A_{i}$ are constants. Besides, not all of $A_{i}$ are zero, otherwise by Proposition 8.1 the tuple $\left(r_{1}, r_{2} \ldots, r_{m}\right)$ is exceptional. Hence, there is a constant $c$ such that $\tau=c t$ is the canonical parameter on the curve $\Lambda$. Then the statement of the lemma follows from the formula (8.1).

Remark 8.3. In the case when $\rho_{1}$ is constant it can be shown that

$$
\begin{aligned}
& \rho_{i} \equiv \frac{\alpha_{m, i}}{\alpha_{m, 1}^{i}}\left(\rho_{1}\right)^{i}, \quad \forall 1 \leq i \leq i_{0}-1, \\
& \rho_{i_{0}} \equiv(-1)^{i_{0}-1} \varepsilon+\frac{\alpha_{m, i_{0}}}{\alpha_{m, 1}^{i_{0}}} \rho_{1}^{i_{0}} .
\end{aligned}
$$


where the constants $\alpha_{m, i}$ are given by formula 1.5. If $\rho_{1}$ is constant and all $\rho_{i}$, with $2 \leq i \leq i_{0}$ satisfy relations (8.5)-(8.6), then the tuple $\left(\rho_{1}, \ldots, \rho_{i_{0}}, \rho_{i_{0}+1}(\tau), \ldots, \rho_{m}(\tau)\right)$ is compatible. Another way to prove Lemma 8.1 is by using this description of the compatible tuples: the required constant $c$ can be found explicitly using formula (8.6).

Finally, taking the $i$ th symplectic curvature for Jacobi curves (parameterized by the canonical parameter) of all abnormal extremals living in $\widetilde{\mathscr{R}}$, we obtain the invariants of the rank 2 distribution $D$, called the its ith symplectic curvature and denoted also by $\rho_{i}$. The symplectic curvatures are scalar valued functions on $\widetilde{\mathscr{R}}$.

\section{The maximally symmetric models}

Now we will find all structures from the considered classes having the pseudo-group of local symmetries of dimension equal to $2 n-3$. As a consequence of Corollary 7.1 if an object from the considered class has the pseudo-group of local symmetries of dimension equal to $2 n-3$ then all structure functions of the canonical frame (7.16) must be constant. Note that formula (8.1) can be rewritten in terms of the canonical frame (7.16) as follows

$$
\left[h, \varepsilon_{2 m}\right]=\sum_{i=1}^{m}(-1)^{i+1}(\operatorname{ad} h)^{m-i}\left(\rho_{i}\left(\operatorname{ad} h^{m-i} \varepsilon_{1}\right) \quad \bmod \operatorname{span}\{\mathrm{e}, \mathrm{h}\}\right.
$$

where $\rho_{i}$ are the $i$ th symplectic curvatures of a structures under consideration. This implies that the symplectic curvatures of all order must be constant for any structure from the considered classes having $2 n-3$-dimensional pseudo-group of local symmetries . This together with Corollary 8.1 and Lemma 8.1 for the case of distributions implies that the following theorem is equivalent to Theorem 1.1

Theorem 9.1. Given any tuples of $n-3$ numbers $\left(r_{1}, \ldots, r_{n-3}\right)$ compatible in the sense of Definition 8.1 there exists the unique, up to local equivalence, rank 2 distribution in $R^{n}$ of maximal class, $n \geq 5$, with at least one non-vanishing generalized Wilczynski invariant such that its group of local symmetries has dimension $2 n-3$ and the ith symplectic curvature is identically equal to $r_{i}$ for any $1 \leq i \leq n-3$. Such distribution is locally equivalent to the distribution $D_{\left(r_{1}, \ldots, r_{n-3}\right)}$ spanned by the vector fields from (1.2)-(1.3).

Proof. Let us prove the uniqueness. Take a structure from the considered class having the pseudogroup of local symmetries of dimension $2 n-3$ and the $i$ th symplectic curvature identically equal to $r_{i}$ for any $1 \leq i \leq m$, where, as before, $m=n-3$. Then, as was already mentioned, all structure functions of the canonical frame (7.16) must be constant. The uniqueness will be proved if we show that all nontrivial structure function (i.e. those that are not prescribed by the normalization conditions for the canonical frame) are uniquely determined by the tuple $\left(r_{1}, \ldots, r_{n-3}\right)$.

Let $\varepsilon_{1}$ be as in the Lemma 7.1. Denote

$$
\varepsilon_{i+1}:=(\operatorname{ad} h)^{i} \varepsilon_{1}, \quad v=\left[\varepsilon_{1}, \varepsilon_{2 m}\right]
$$

In this notations the canonical frame (7.16) is $\left\{e, h, \varepsilon_{1}, \ldots, \varepsilon_{2 m}, \eta\right\}$. 
(1) Let us prove that

$$
\left[e, \varepsilon_{1}\right]=-\frac{1}{2} \varepsilon_{1}
$$

where, as before $e$ is the Euler field. Indeed, from (7.14)

$$
\left[e, \varepsilon_{1}\right]=-\frac{1}{2} \varepsilon_{1}+a e
$$

where $a$ is constant by our assumptions. Then, using the Jacobi identity and the fact that

$$
[e, h]=0
$$

we get that

$$
\left[e, \varepsilon_{2}\right]=\left[e,\left[h, \varepsilon_{1}\right]\right]=\left[h,\left[e, \varepsilon_{1}\right]\right]=\left[h,-\frac{1}{2} \varepsilon_{1}+a e\right]=-\frac{1}{2} \varepsilon_{2}
$$

Further, from the normalization condition (7.10) and formula (9.4) it follows that

$$
\left[e,\left[\varepsilon_{1}, \varepsilon_{2}\right]\right] \in \operatorname{span}\left\{e(\lambda), h(\lambda), \varepsilon_{1}(\lambda)\right\}
$$

On the other hand, using the Jacobi identity and formulas (9.4),(9.5),(9.6), we get that

$$
\begin{aligned}
& {\left[e,\left[\varepsilon_{1}, \varepsilon_{2}\right]\right]=\left[\left[e, \varepsilon_{1}\right], \varepsilon_{2}\right]+\left[\varepsilon_{1},\left[e, \varepsilon_{2}\right]\right]=\left[-\frac{1}{2} \varepsilon_{1}+a e, \varepsilon_{2}\right]-} \\
& \frac{1}{2}\left[\varepsilon_{1}, \varepsilon_{2}\right] \equiv-\frac{a}{2} \varepsilon_{2} \bmod \operatorname{span}\left\{e(\lambda), h(\lambda), \varepsilon_{1}(\lambda)\right\}
\end{aligned}
$$

which together with (9.7) implies that $a=0$.

(2) By analogy with the chain of the equalities (9.7) we can prove that

$$
\left[e, \varepsilon_{i}\right]=-\frac{1}{2} \varepsilon_{i}, \quad \forall 1 \leq i \leq 2 m,
$$

which in turn implies by the Jacobi identity that

$$
\left[e,\left[\varepsilon_{i}, \varepsilon_{j}\right]\right]=-\left[\varepsilon_{i}, \varepsilon_{j}\right], \quad \forall 1 \leq i, j \leq 2 m .
$$

In particular, $[e, \eta]=-\eta$.

(3) Let us show that

$$
\left[h, \varepsilon_{2 m}\right]=\sum_{i=1}^{m-1}(-1)^{i+1} r_{i} \varepsilon_{2(m-i)}
$$

From (9.1) and our assumptions it follows that

$$
\left[h, \varepsilon_{2 m}\right]=\sum_{i=1}^{m-1}(-1)^{i+1} r_{i} \varepsilon_{2(m-i)}+\gamma e+\delta h
$$

for some constants $\gamma$ and $\delta$. Applying ad $e$ to both sides of (9.11) and using the Jacobi identity and formulas (9.5) and (9.8), we will get that $\gamma=\delta=0$, which implies (9.11). 
(4) Let us prove that

$$
\left[\varepsilon_{i}, \varepsilon_{j}\right]=d_{i j} \eta
$$

for some constants $d_{i j}$ Indeed, in general

$$
\left[\varepsilon_{i}, \varepsilon_{j}\right]=b_{i j} e+c_{i j} h+d_{i j} \eta+\sum_{k=1}^{2 m} a_{i j}^{k} \varepsilon_{k}
$$

where $a_{i j}^{k}, b_{i j}, c_{i j}$ and $d_{i j}$ are constant by our assumptions. Applying ade to both sides of (9.13) and using the Jacobi identity and the formulas (9.5), (9.8), and (9.9), we get

$$
-\left[\varepsilon_{i}, \varepsilon_{j}\right]=-d_{i j} \eta-\frac{1}{2} \sum_{k=1}^{2 m} a_{i j}^{k} \varepsilon_{k}
$$

Comparing (9.13) and (9.14) we get that $a_{i j}^{k}=b_{i j}=c_{i j}=0$, which implies (9.12).

(5) Moreover, by Remark 8.1 and the definition of the vector field $\eta$ (see (9.2)) the constants $d_{i j}$ from (9.12) are either identically equal to 0 , if $i+j<2 m$ or equal to $(-1)^{i-1}$, if $i+$ $j=2 m+1$, or they are polynomial expressions (with universal constant coefficients) with respect to the constant symplectic curvatures $r_{1} \ldots, r_{m}$, if $i+j>2 m$.

(6) The remaining brackets of the canonical frame are obtained iteratively from the brackets considered in the previous items.

Therefore all nontrivial structure functions of the canonical frame are determined by the tuple $\left(r_{1}, \ldots, r_{n-3}\right)$, which completes the proof of uniqueness.

To prove the existence one checks by the direct computations that the models $D_{\left(r_{1}, \ldots, r_{m}\right)}$ have the prescribed symplectic curvatures and that all structure functions of their canonical frame are constant similarly to the proof of the existence part of Theorem 3 in [11], devoted to the computation of the canonical frame for $D_{(0, \ldots, 0)}$.

\section{References}

[1] A.A. Agrachev, Feedback-invariant optimal control theory - II. Jacobi Curves for Singular Extremals, J. Dynamical and Control Systems, 4 (1998), No. 4 , 583-604.

[2] A.A. Agrachev, R.V. Gamkrelidze, Feedback-invariant optimal control theory - I. Regular extremals, J. Dynamical and Control Systems, 3 (1997), No. 3, 343-389.

[3] A. Agrachev, I. Zelenko, Geometry of Jacobi curves. I, J. Dynamical and Control systems, 8 (2002), No. 1, 93-140.

[4] A. Agrachev, I. Zelenko, Nurowski's conformal structures for (2,5)-distributions via dynamics of abnormal extremals, Proceedings of RIMS Symposium on "Developments of Cartan Geometry and Related Mathematical Problems", "RIMS Kokyuroku” series 1502, 204-218, arxiv math.DG/0605059.

[5] A. Agrachev, I. Zelenko, On feedback classification of control-affine systems with one and twodimensional inputs, SIAM Journal on Control and Optimization, 46 (2007), Issue 4, 1431-1460.

[6] D.V. Alekseevsky, A. Spiro, Prolongations of Tanaka structures and regular CR structures, Selected topics in Cauchy-Riemann geometry, 1-37, Quad. Mat., 9, Dept. Math., Seconda Univ. Napoli, Caserta, 2001.

[7] I. Anderson, B. Kruglikov, Rank 2 distributions of Monge equations: symmetries, equivalences, extensions, Advances in Mathematics Volume 228, Issue 3, 2011, 1435-1465.

[8] E. Cartan, Les systemes de Pfaff a cinq variables et les equations aux derivees partielles du second ordre, Oeuvres completes, Partie II, vol.2, Paris, Gautier-Villars, 1953, 927-1010. 
[9] B. Doubrov, Generalized Wilczynski invariants for non-linear ordinary differential equations, In: Symmetries and Overdetermined Systems of Partial Differetial Equations, IMA Volume 144, Springer, NY, 2008, pp. 25-40.

[10] B. Doubrov, I. Zelenko, A canonical frame for nonholonomic rank two distributions of maximal class, C.R. Acad. Sci. Paris, Ser. I, 342, (2006), Issue 8, 589-594.

[11] B. Doubrov, I. Zelenko, On local geometry of nonholonomic rank 2 distributions, Journal of London Mathematical Society, (2) 80 (2009), No. 3, 545-566.

[12] B. Doubrov, I. Zelenko, Equivalence of variational problems of higher order, Differential Geometry and its Applications, 29 (2011), 255-270.

[13] B. Doubrov, I. Zelenko, On local geometry of rank 3 distributions with 6-dimensional square, preprint 2008, arXiv 0807.3267v1[math. DG], 40 pages.

[14] B. Doubrov, I. Zelenko, Geometry of curves in generalized flag varieties, Transformation Groups, 18 (2013), Issue 2, 361-383.

[15] B. Doubrov, I. Zelenko, On geometry of curves of flags of constant type, Central European J. Math., 10 (2012), No. 5, 1836-1871.

[16] B. Doubrov, I. Zelenko, On geometry of affine control systems with one input, in the book "Geometric Control Theory and sub-Riemannain Geometry", G. Stefani, U. Boscain, M. Sigalotti, J.-P. Gauthier, A. Sarychev (Eds.), Springer INdAM series, Vol. 7, to appear in 2014.

[17] W. Fulton, J. Harris, Representation theory: a first course, Springer-Verlag, NY, 1991.

[18] B. Kruglikov, The gap phenomenon in the dimension study of finite type systems. Central European J. Math. 10 (2012), No. 5, 1605-1618.

[19] B. Kruglikov, D. The, The gap phenomenon in parabolic geometries, preprint 2013, arXiv 1303.130v3[math.DG], 54 pages.

[20] E. A. Kwessi Nyandjou, Generalized Sturm Theorem for self-adjoint differential operators of higher order, diploma thesis in the Diploma program of ICTP- International Center for Theoretical Physics, Trieste, Italy 2006/2007 (under supervision of I. Zelenko).

[21] M. A. Naimark, Linear Differential Operators. Part I: Elementary theory of linear differential operators with additional material by the author, New York: Frederick Ungar Publishing Co. XIII (1967), 144 pages.

[22] P. Nurowski, Differential equations and conformal structures, J. Geom. Phys. 55 (2005), No. 1, 19-49.

[23] P.J. Olver, Symmetry, invariants, and equivalence, New York: Springer-Verlag, 1995.

[24] N. Tanaka, On differential systems, graded Lie algebras and pseudo-groups, J. Math. Kyoto. Univ., 10 (1970), 1-82.

[25] E.J. Wilczynski, Projective differential geometry of curves and ruled surfaces, Teubner, Leipzig, 1905.

[26] I. Zelenko, Nonregular abnormal extremals of 2-distribution: existence, second variation and rigidity, J. Dynamical and Control systems , 5 (1999), No. 3, 347-383.

[27] I. Zelenko, On Variational Approach to Differential Invariants of Rank 2 Vector Distributions, Differential Geometry and Its Applications, Vol. 24, Issue 3 (May 2006), 235-259.

[28] I. Zelenko, Fundamental form and the Cartan tensor of (2,5)-distributions coincide, J. Dynamical and Control Systems, Vol.12, No. 2, April 2006, 247-276

[29] I. Zelenko, Complete systems of invariants for rank 1 curves in Lagrange Grassmannians, Differential Geom. Application, Proc. Conf. Prague, 2005, pp 365-379, Charles University, Prague, arxiv math. DG/0411190.

[30] I. Zelenko, On Tanaka's prolongation procedure for filtered structures of constant type, Symmetry, Integrability and Geometry: Methods and Applications (SIGMA), Special Issue "Elie Cartan and Differential Geometry", 5, (2009), 21 pages.

[31] I. Zelenko, Invariants of curves in the Lagrange Grassmannian and differential geometry of smooth control systems, $\mathrm{PhD}$ Thesis, Technion, Israel, 2002, (under supervision of A. Agrachev and A. Ioffe). 